\title{
Multi objective optimization of PMEDM using response surface methodology coupled with fuzzy based desirability function approach
}

\author{
Munmun Bhaumik $^{\mathbf{a}^{*}}$ and Kalipada Maity
}

${ }^{a}$ Department of Mechanical Engineering, NIT Rourkela, Orissa, India

${ }^{b}$ Department of Mechanical Engineering, NIT Rourkela, Orissa, India

\section{H R O N I C L E}

\begin{tabular}{l}
\hline Article history: \\
Received October 3, 2016 \\
Received in revised format: \\
October 22, 2016 \\
Accepted February 2, 2017 \\
Available online \\
February 22017 \\
\hline Keywords: \\
Desirability \\
Fuzzy-logic method \\
Material removal rate \\
Powder mixed electro discharge \\
machining \\
Surface roughness \\
Tool wear rate
\end{tabular}

Tool wear rate

\section{A B S T R A C T}

Powder mixed electro discharge machining (PMEDM) is a hybrid machining process where the electrically conductive powder is mixed into the dielectric fluid to enhance the machining efficiency as well as surface finish. In this investigation, PMEDM is performed for the machining of AISI 304 stainless steel when silicon carbide powder is mixed into the kerosene dielectric. Peak current, pulse on time, gap voltage, duty cycle and powder concentration are considered as process parameter while material removal rate (MRR), tool wear rate (TWR) and surface roughness $(\mathrm{Ra})$ are considered as response. A face centered central composite design (FCCCD) based response surface methodology (RSM) is applied to design the experiment. A hybrid optimization technique like desirability coupled with fuzzy-logic method is performed to get the optimum level of the multiple performance characteristics. Analysis of variance (ANOVA) is performed for the statistical analysis. The result shows that peak current is the most significant parameter for MRR, TWR and Ra. The optimal setting for maximum MRR, minimum TWR and Ra have been obtained by desirability coupled with fuzzy-logic method.

\section{Introduction}

Among all the nonconventional machining process, electro discharge machining (EDM) is the most popular and successful one. This process is based on an electro-thermal process where electrical energy generates the spark, and thermal energy removes the molten metal from the workpiece. EDM is mainly used to cut the difficult-to-machine material, high strength and high-temperature resistance material (Snoeys et al., 1986). Conventional EDM has some advantages as well as some disadvantages. Therefore, to overcome the disadvantages, hybrid EDM is introduced and powder mixed EDM (PMEDM) is one of the finest hybrid EDM techniques used by the researchers (Rajagopal et al., 2013; Pandey \& Singh, 2010). Dewangan et al. (2015) performed multi-objective optimization to determine

* Corresponding author

E-mail address: munmunbhaumik@gmail.com (M. Bhaumik) 
the optimal parameter setting for improving the surface integrity of AISI P20 tool steel. They used grey relational analysis (GRA) in combination with fuzzy logic to determine the grey fuzzy reasoning grade (GFRG). Again, they adopted the Fuzzy TOPSIS and sensitivity analysis to optimize the surface crack density (SCD), white layer thickness (WLT), surface roughness (SR), and overcut (OC). They found an optimal parametric combination of peak current $=1 \mathrm{~A}$, pulse on time $=10 \mu \mathrm{s}$, tool-work time $=0.2 \mathrm{~s}$, tool lift time $=1.5 \mathrm{~s}$ to reduce the surface integrity as well as the dimensional accuracy (Dewangan et al., 2015). Pandey and Panda (2015) adopted the Taguchi methodology in combination with fuzzylogic based on desirability function to optimize the bone drilling process to minimize the drilling induced damage bone. Bhaumik and Maity (2014, 2015, and 2016) studied the effect of tungsten carbide electrode on the machining performance of EDM during machining of AISI 304 and optimizing the responses viz. material removal rate (MRR), tool wear rate (TWR), surface roughness (Ra) using desirability coupled with grey relational analysis (GRA).

Singh and Yeh (2012) performed grey relational analysis to appraise the efficiency of optimizing multiple performance characteristics of abrasive powder mixed EDM (APM-EDM) of $6061 \mathrm{Al} / \mathrm{Al}_{2} \mathrm{O}_{3 \mathrm{p}} / 20_{\mathrm{p}}$. Tripathy and Tripathy (2016) applied Taguchi method coupled with technique for order of preference by similarity to ideal solution (TOPSIS) and GRA to evaluate the effectiveness of multiple performance characteristics viz. MRR, TWR, $\mathrm{R}_{\mathrm{a}}$ for PMEDM during machining of $\mathrm{H}-11$ die steel. Reddy et al. (2015) performed multi objective optimization for the response parameters such as MRR, TWR and surface roughness (SR) based on Taguchi methodology coupled with GRA for PH174 stainless steel. Shivakoti et al. (2013) reported the influence of $\mathrm{NaNO}_{3}$ mixed water during machining of D3 die steel during powder mixed electro discharge machining. They applied GRA to optimize the multi-performance characteristics such as MRR, TWR and overcut (OC). Tang and Du (2014) adopted Taguchi methodology in combination with GRA during green electrical discharge machining of Ti$6 \mathrm{Al}-4 \mathrm{~V}$. They reported that this optimization was efficient and effective for the optimization of multi performance characteristics. Rupajati et al. (2014) adopted the Taguchi method coupled with fuzzylogic method to optimize the multiple responses viz. recast layer thickness and surface roughness. They found that this optimization technique significantly could improve the multiple responses. The same optimization technique was applied to predict the MRR, TWR and SR in ultrasonic-assisted EDM (USEDM) (Shabgarda et al., 2013). Another manufacturing processes also have been optimized using similar types of optimization technique (Barzani et al., 2015; Pandey \& Dubey, 2012; Acilar \& Arslan, 2011).

From the above literature review, it is evident that hybrid optimization technique has mostly been adopted in EDM processes. In this study, hybrid optimization technique (Response surface methodology and desirability coupled with fuzzy-logic method) is discussed for determining the optimum level of machining parameter for improving the MRR and lower down the TWR and $\mathrm{R}_{\mathrm{a}}$ simultaneously.

\section{Material and Methods}

In this investigation, powder mixed electro discharge machining (PMEDM) is carried out considering AISI 304 stainless steel and tungsten carbide $(\varphi 10 \mathrm{~mm})$ as workpiece and tool material, respectively. In this study, $\mathrm{SiC}$ powder $(\sim 30 \mu \mathrm{m})$ is mixed into the kerosene oil (dielectric constant=2, electrical conductivity $=1.6 \times 10^{-14} \mathrm{~S} / \mathrm{m}$ ). Experiments are carried out in Electronica-Electraplus PS $50 \mathrm{ZNC}$ machine. The chemical composition of AISI 304 stainless steel is given in Table 1.

The machining parameters considered in this study are i) peak current $\left(\mathrm{I}_{\mathrm{p}}\right)$, ii) pulse on time $\left(\mathrm{T}_{\text {on }}\right)$, iii) gap voltage $\left(\mathrm{V}_{\mathrm{g}}\right)$, iv) duty cycle $(\mathrm{r})$, v) powder concentration $\left(\mathrm{P}_{\mathrm{C}}\right)$ while (i) material removal rate (MRR) $\left(\mathrm{mm}^{3} \mathrm{~min}^{-1}\right)$, (ii) tool wear rate $(\mathrm{TWR})\left(\mathrm{mm}^{3} \mathrm{~min}^{-1}\right)$ and (iii) surface roughness $\left(\mathrm{R}_{\mathrm{a}}\right)(\mu \mathrm{m})$ are considered as response. The machining parameters and their levels are tabulated in Table 2 . A face-centered central composite design (FCCCD) based response surface methodology (RSM) has been adopted to design 
the PMEDM process. RSM is a group of mathematical and statistical techniques which are used for modeling and analysis of problems where the response is controlled by the input variables, and the objective is to develop a relationship amongst them (Montgomery, 2001). A total of 33 experiments were performed for this study. The experimental layout, result and their respective Desirability fuzzy reasoning grade (DFRG) values are tabulated in Table 3.

\section{Table 1}

Chemical Composition of AISI 304

\begin{tabular}{lllllllll}
\hline Element & $\mathrm{C}$ & $\mathrm{Mn}$ & $\mathrm{Si}$ & $\mathrm{P}$ & $\mathrm{S}$ & $\mathrm{Cr}$ & $\mathrm{Ni}$ & $\mathrm{Fe}$ \\
\hline$\%$ & 0.06 & 0.72 & 1.26 & 0.05 & 0.11 & 16.36 & 6.95 & Balance \\
\hline
\end{tabular}

\section{Table 2}

Level values of machining parameter

\begin{tabular}{llcc}
\hline S1. No. & Factors & \multicolumn{2}{c}{ Levels } \\
\cline { 3 - 4 } & & Low $(-1)$ & High $(+1)$ \\
\hline 1 & $\mathrm{I}_{\mathrm{P}}(\mathrm{A})$ & 4 & 8 \\
2 & $\mathrm{~T}_{\mathrm{on}}(\mu \mathrm{s})$ & 50 & 65 \\
3 & $\mathrm{~V}_{\mathrm{g}}(\mathrm{V})$ & 45 & 65 \\
4 & $\mathrm{I}(\%)$ & 55 & 10 \\
5 & $\mathrm{P}_{\mathrm{C}}(\mathrm{g} / \mathrm{l})$ & 0 & \\
6 & Polarity & \multicolumn{2}{c}{} \\
7 & Machining Time (min) & Kerosene Oil \\
8 & Dielectric Fluid & \multicolumn{2}{c}{ Ke } \\
\hline
\end{tabular}

\subsection{Experimental Procedure}

Experiments are performed according to design matrix. During machining, material removal from the workpiece and electrode are measured using the weight loss method and then converted to volumetric material loss. The average surface roughness is measured by Talysurf (Model: Taylor Hobson, Surtronic 3+). The output responses such as MRR and TWR are calculated from the experimental data.

$$
\text { Material Re moval Rate }(M R R)=\frac{\text { Volumetric material loss from workpiece }\left(\mathrm{mm}^{3}\right)}{\text { Machining Time }(\mathrm{min})}
$$

\section{Result and discussion}

\subsection{Optimization using desirability with the fuzzy-logic method}

Multi response optimization converts all the output responses to a scale-free single response and then optimizes the single response. In this study, a hybrid technique such as desirability coupled with the fuzzy-logic method is used to optimize the responses.

\subsubsection{Steps for desirability-fuzzy logic method}

1. Calculate the desirability index $\left(\mathrm{d}_{\mathrm{i}}\right)$ for each output response.

2. Apply fuzzy-logic system. The fuzzifier uses the membership function to fuzzify the desirability of each performance characteristics.

3. Generate the fuzzy rules. Then deffuzzifier converts the predicted fuzzy value into desirability-fuzzy reasoning grade (DFRG).

4. Perform the statistical analysis of variance (ANOVA). 
5. Evaluate the optimal parameter settings for the machining parameter.

6. Perform the confirmation test

\subsubsection{Calculation of Desirability function}

To calculate the desirability index $\left(\mathrm{d}_{\mathrm{i}}\right)$ for each output response, higher the better is chosen for material removal rate and lower the better is chosen for tool wear rate and surface roughness. The desirability index value of material removal rate, tool wear rate and surface roughness are calculated by Eq. (4) and Eq. (7) respectively.

Desirability for "Higher-the-better" (HB) (Ramanujam et al., 2014)

$$
\begin{aligned}
& \text { If } \hat{y} \leq y_{\min }, d_{i}=0 \\
& \text { If, } y_{\min } \leq \hat{y} \leq y_{\max }, d_{i}=\left(\frac{\hat{y}-y_{\min }}{y_{\max }-y_{\min }}\right)^{r} \\
& \text { If } \hat{y} \geq y_{\text {max }}, d_{i}=1 \\
& \text { Desirability for "Lower-the-better" (LB) } \\
& \text { If, } \hat{y} \leq y_{\min }, d_{i}=1 \\
& \text { If } y_{\min } \leq \hat{y} \leq y_{\text {max }}, d_{i}=\left(\frac{\hat{y}-y_{\max }}{y_{\min }-y_{\max }}\right)^{r} \\
& \text { If, } \hat{y} \geq y_{\text {max }}, d_{i}=0
\end{aligned}
$$

where, $y_{\min }$ is a lower tolerance limit of $\hat{y}, y_{\max }$ is a higher tolerance limit of $\hat{y}, d_{i}$ is desirability value, $r$ represents the desirability function index. In this study, it is considered as 1 .

\subsubsection{Desirability fuzzy-logic method}

In desirability method, each response is categorized by higher the better, lower the better and nominal is the best quality characteristics. A complicated multi-objective optimization problem can be solved by integrating the desirability analysis and fuzzy-logic method.

Now a day's fuzzy rule based modeling is widely used because it can easily represent the uncertain and imprecise relationships which is very difficult to describe with the precise mathematical modeling (Latha \& Senthilkumar, 2009, 2010; Singh et al., 2013). A fuzzy logic system consists of a fuzzifier, membership functions, a fuzzy rule base, an interference engine and a defuzzifier. Different types of membership functions are used for fuzzy analysis such as triangular, trapezoidal, sigmoid and Gaussian, etc. The most popular fuzzy interferences are Mamdani and Sugeno. Mamdani model has been widely used to solve the complicated problems because of its easiness (Latha \& Senthilkumar, 2009, 2010; Singh et al., 2013). For this study, a triangular shaped membership function is used for fuzzification of input and output function.

For fuzzifying, the input variable, the linguistic membership function such as small, medium and large are used. The output is fuzzified by using the membership function such as very small, small, medium, large and very large. 33 fuzzy rules are used for fuzzy interface engine based on 33 experiments run. The crisp value of DFRG is obtained using the center of gravity defuzzification method. MATLAB fuzzy logic toolbox is used to implementing the fuzzy logic. The relationships between the input and the output are presented in the form of 'if-then' rule which are presented below:

Rule 1: if MRR is large and TWR is large, and $\mathrm{R}_{\mathrm{a}}$ is large then DFRG is very large Rule 2: if MRR is medium and TWR is medium, and $R_{a}$ is medium then DFRG is large 
Rule $n$ : if MRR is small and TWR is small, and $R_{a}$ is small then DFRG is very small

Fuzzy rules are directly derived based on the larger the better characteristics. The membership functions of three input and output parameters are shown in Fig.1 and Fig. 2. Fuzzy logic rule viewer is shown in Fig.3. In this figure the row represents the fuzzy rules, the first three columns represent the input desirability of MRR, TWR and $R_{a}$ respectively. The last column represents the defuzzified multi characteristics perform index i.e. DFRG. The desirability and DFRG values are tabulated in Table 3.

\section{Table 3}

Experimental layout and DFRG value of PMEDM performance characteristics

\begin{tabular}{|c|c|c|c|c|c|c|c|c|c|c|c|c|}
\hline \multirow{2}{*}{$\begin{array}{l}\text { Exp. } \\
\text { No. }\end{array}$} & \multicolumn{5}{|c|}{ Process parameter } & \multicolumn{3}{|c|}{ Observed value } & \multicolumn{2}{|c|}{ desirability } & \multirow[b]{2}{*}{$\mathrm{d}_{\mathrm{i}}\left(\mathrm{R}_{\mathrm{a}}\right)$} & \multirow[b]{2}{*}{ DFRG } \\
\hline & $\mathrm{I}_{\mathrm{p}}$ & $\mathrm{T}_{\text {on }}$ & $\mathrm{V}_{\mathrm{g}}$ & r & $\mathrm{P}_{\mathrm{C}}$ & MRR & TWR & $\mathrm{R}_{\mathrm{a}}$ & $\mathrm{d}_{\mathrm{i}}(\mathrm{MRR})$ & $\mathrm{d}_{\mathrm{i}}(\mathrm{TWR})$ & & \\
\hline 1 & 4 & 50 & 45 & 55 & 10 & 1.6458 & 0.02132 & 5.640 & 0.156322 & 0.833307 & 1 & 0.594 \\
\hline 2 & 8 & 50 & 45 & 55 & 0 & 4.1650 & 0.05632 & 8.390 & 0.541235 & 0.5 & 0 & 0.395 \\
\hline 3 & 4 & 150 & 45 & 55 & 0 & 2.5791 & 0.02132 & 6.730 & 0.156737 & 1 & 0.7575 & 0.536 \\
\hline 4 & 8 & 150 & 45 & 55 & 10 & 6.6295 & 0.07096 & 7.980 & 0.725261 & 0.749922 & 0.0675 & 0.441 \\
\hline 5 & 4 & 50 & 65 & 55 & 0 & 1.2083 & 0.02132 & 6.450 & 0.085122 & 0.916693 & 0.85 & 0.451 \\
\hline 6 & 8 & 50 & 65 & 55 & 10 & 6.1375 & 0.03399 & 7.780 & 0.812682 & 0.5 & 0.565 & 0.279 \\
\hline 7 & 4 & 150 & 65 & 55 & 10 & 2.3041 & 0.01066 & 5.810 & 0.096015 & 0.916693 & 0.825 & 0.464 \\
\hline 8 & 8 & 150 & 65 & 55 & 0 & 6.3583 & 0.09396 & 8.672 & 0.846191 & 0.333307 & 0.0475 & 0.485 \\
\hline 9 & 4 & 50 & 45 & 65 & 0 & 2.1366 & 0.03398 & 6.380 & 0.024299 & 0.5 & 0.9675 & 0.080 \\
\hline 10 & 8 & 50 & 45 & 65 & 10 & 5.5041 & 0.05131 & 7.340 & 0.812167 & 0.416693 & 0.2 & 0.561 \\
\hline 11 & 4 & 150 & 45 & 65 & 10 & 2.1458 & 0.02132 & 6.210 & 0.3652 & 0.916693 & 0.975 & 0.619 \\
\hline 12 & 8 & 150 & 45 & 65 & 0 & 5.7860 & 0.09396 & 8.340 & 0.888594 & 0 & 0.0925 & 0.250 \\
\hline 13 & 4 & 50 & 65 & 65 & 10 & 2.2250 & 0.02132 & 5.800 & 0 & 1 & 0.8575 & 0.665 \\
\hline 14 & 8 & 50 & 65 & 65 & 0 & 5.1291 & 0.08123 & 8.370 & 0.848289 & 0.5 & 0.1175 & 0.493 \\
\hline 15 & 4 & 150 & 65 & 65 & 0 & 1.4668 & 0.03632 & 6.910 & 0.042617 & 0.833307 & 0.7175 & 0.863 \\
\hline 16 & 8 & 150 & 65 & 65 & 10 & 8.0125 & 0.09686 & 7.240 & 1 & 0.083307 & 0.4325 & 0.472 \\
\hline 17 & 6 & 100 & 55 & 60 & 5 & 3.6664 & 0.04265 & 7.110 & 0.412001 & 0.75 & 0.485 & 0.547 \\
\hline 18 & 6 & 100 & 55 & 60 & 5 & 3.4683 & 0.04265 & 7.020 & 0.411687 & 0.75 & 0.4425 & 0.5 \\
\hline 19 & 6 & 100 & 55 & 60 & 5 & 3.6666 & 0.03865 & 7.130 & 0.415343 & 0.612066 & 0.4325 & 0.485 \\
\hline 20 & 6 & 100 & 55 & 60 & 5 & 3.5208 & 0.04265 & 7.020 & 0.424377 & 0.662316 & 0.4675 & 0.5 \\
\hline 21 & 6 & 100 & 55 & 60 & 5 & 3.5591 & 0.03998 & 7.070 & 0.409539 & 0.612066 & 0.4187 & 0.5 \\
\hline 22 & 6 & 100 & 55 & 60 & 5 & 3.5015 & 0.03965 & 7.110 & 0.394361 & 0.68748 & 0.4425 & 0.493 \\
\hline 23 & 4 & 100 & 55 & 60 & 5 & 2.3708 & 0.03198 & 6.330 & 0.123228 & 0.68748 & 0.9325 & 0.537 \\
\hline 24 & 8 & 100 & 55 & 60 & 5 & 6.1910 & 0.07397 & 8.150 & 0.796462 & 0.166693 & 0.2425 & 0.433 \\
\hline 25 & 6 & 50 & 55 & 60 & 5 & 2.8900 & 0.03632 & 6.260 & 0.402779 & 0.778603 & 0.5225 & 0.426 \\
\hline 26 & 6 & 150 & 55 & 60 & 5 & 3.6875 & 0.05631 & 6.630 & 0.555018 & 0.61191 & 0.3925 & 0.5 \\
\hline 27 & 6 & 100 & 45 & 60 & 5 & 3.2667 & 0.03398 & 6.940 & 0.425093 & 0.662316 & 0.4 & 0.445 \\
\hline 28 & 6 & 100 & 65 & 60 & 5 & 3.6830 & 0.03631 & 7.060 & 0.407491 & 0.662316 & 0.4625 & 0.5 \\
\hline 29 & 6 & 100 & 55 & 55 & 5 & 3.2583 & 0.03198 & 7.220 & 0.377023 & 0.833307 & 0.3925 & 0.475 \\
\hline 30 & 6 & 100 & 55 & 65 & 5 & 3.5166 & 0.04065 & 7.270 & 0.457232 & 0.662316 & 0.3825 & 0.5 \\
\hline 31 & 6 & 100 & 55 & 60 & 0 & 2.8910 & 0.05331 & 7.740 & 0.292115 & 0.611988 & 0.3 & 0.44 \\
\hline 32 & 6 & 100 & 55 & 60 & 10 & 3.9583 & 0.03298 & 6.970 & 0.387074 & 0.833307 & 0.6175 & 0.550 \\
\hline 33 & 6 & 100 & 55 & 60 & 5 & 3.6333 & 0.04365 & 7.060 & 0.383418 & 0.612066 & 0.4175 & 0.514 \\
\hline
\end{tabular}



Fig. 1. Membership functions for MRR, TWR and $\mathrm{R}_{\mathrm{a}}$

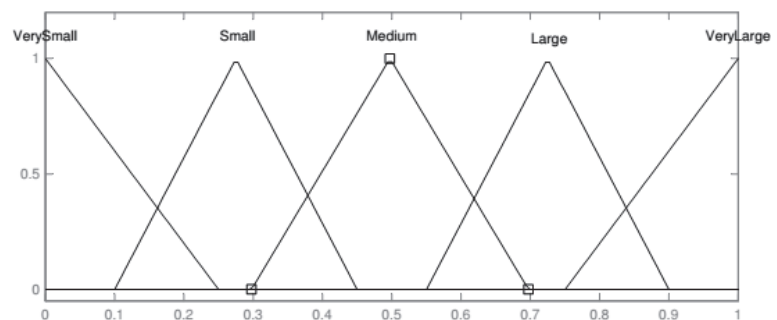

Fig. 2. Membership functions for desirabilityfuzzy reasoning grade 


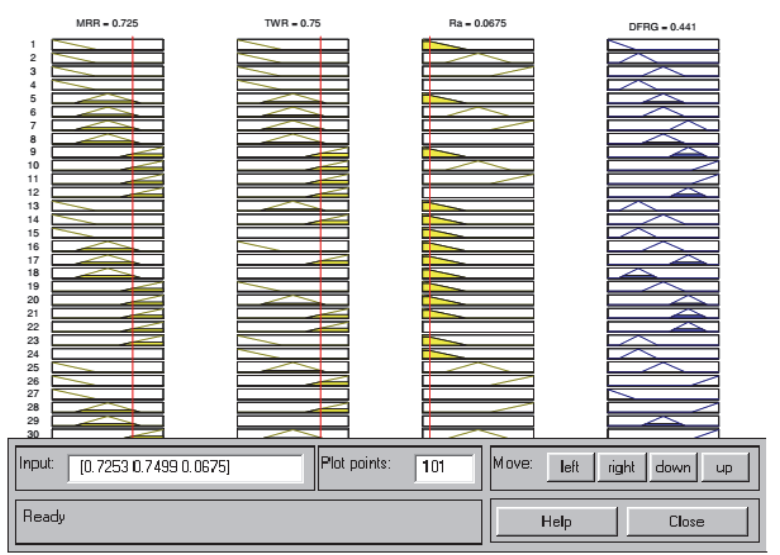

Fig. 3. Fuzzy logic reasoning procedure

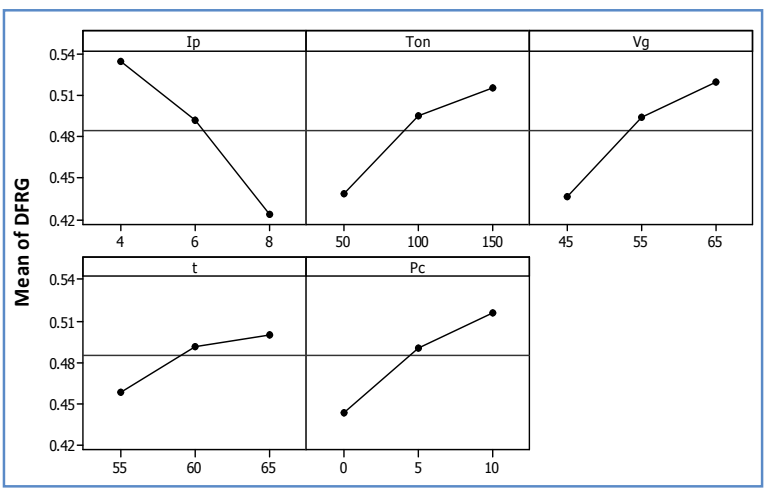

Fig. 4. Main effect plot for DFRG

Table 4

ANOVA table for DFRG

\begin{tabular}{|c|c|c|c|c|c|c|}
\hline Source & $\mathrm{DF}$ & Seq SS & Adj MS & $\mathrm{F}$ & $\mathrm{P}$ & $\%$ of contribution \\
\hline Regression & 11 & 0.490514 & 0.044592 & 50.29 & 0.000 & 96.34 \\
\hline Linear & 5 & 0.144760 & 0.052045 & 58.69 & 0.000 & 28.43 \\
\hline IP & 1 & 0.055411 & 0.022707 & 25.61 & 0.000 & 10.88 \\
\hline $\mathrm{T}_{\text {on }}$ & 1 & 0.026106 & 0.080378 & 90.64 & 0.000 & 5.12 \\
\hline $\mathrm{V}_{\mathrm{g}}$ & 1 & 0.031375 & 0.054308 & 61.24 & 0.000 & 6.16 \\
\hline$\Gamma$ & 1 & 0.008171 & 0.105219 & 118.65 & 0.000 & 1.60 \\
\hline $\mathrm{Pc}$ & 1 & 0.023697 & 0.000417 & 0.47 & $0.500 *$ & 4.65 \\
\hline Interaction & 6 & 0.345754 & 0.057626 & 64.98 & 0.000 & 67.90 \\
\hline $\mathrm{I}_{\mathrm{P}} \times \mathrm{T}_{\text {on }}$ & 1 & 0.037201 & 0.037201 & 41.95 & 0.000 & 7.30 \\
\hline $\mathrm{I}_{\mathrm{P}} \times \mathrm{V}_{\mathrm{g}}$ & 1 & 0.017589 & 0.017589 & 19.84 & 0.000 & 3.45 \\
\hline $\mathrm{T}_{\text {on }} \times \mathrm{Pc}$ & 1 & 0.041851 & 0.041851 & 47.19 & 0.000 & 8.22 \\
\hline $\mathrm{V}_{\mathrm{g}} \times_{\Gamma}$ & 1 & 0.100600 & 0.100600 & 113.45 & 0.000 & 19.75 \\
\hline $\mathrm{V}_{\mathrm{g}} \times \mathrm{Pc}$ & 1 & 0.116230 & 0.116230 & 131.07 & 0.000 & 22.82 \\
\hline$\Gamma \times \mathrm{Pc}$ & 1 & 0.032283 & 0.032283 & 36.41 & 0.000 & 6.34 \\
\hline Residual Error & 21 & 0.018622 & 0.000887 & & & 3.65 \\
\hline Lack-of-Fit & 15 & 0.016146 & 0.001076 & 2.61 & $0.122 *$ & 3.17 \\
\hline Pure Error & 6 & 0.002476 & 0.000413 & & & 0.48 \\
\hline Total & 32 & 0.509136 & & & & 100 \\
\hline
\end{tabular}

The optimal value for the machining parameter is shown in Fig.4. From the main effect plot of DFRG, the optimal parametric combination of higher MRR, lower TWR and $R_{a}$ has been found at $I_{P}=4 A$, $\mathrm{T}_{\text {on }}=150 \mu \mathrm{s}, \mathrm{V}_{\mathrm{g}}=65 \mathrm{~V}, \Gamma=65 \%$ and $\mathrm{Pc}=10 \mathrm{~g} / \mathrm{l}$. Statistical analysis of variance (ANOVA) is performed for each machining parameter shown in Table 4. In this table, the insignificant terms are eliminated, and the remaining terms are provided. The regression equation for this model is

$\mathrm{DFRG}=4.41842+0.11166 \times \mathrm{I}_{\mathrm{P}}+0.00468 \times \mathrm{T}_{\text {on }}-0.07251 \times \mathrm{V}_{\mathrm{g}}-0.09195 \times \Gamma+0.01366 \times \mathrm{Pc}-0.00048$ $\mathrm{I}_{\mathrm{P}} \times \mathrm{T}_{\text {on }}-0.00166 \mathrm{I}_{\mathrm{P}} \times \mathrm{V}_{\mathrm{g}}-0.00020 \mathrm{~T}_{\text {on }} \times \mathrm{Pc}+0.00159 \mathrm{~V}_{\mathrm{g}} \times \Gamma-0.00170 \mathrm{~V}_{\mathrm{g}} \times \mathrm{Pc}+0.00180 \Gamma \times \mathrm{Pc}$

\section{Confirmation test}

The confirmation experiment is the ultimate step to establish and confirm the progress of the quality characteristics using the optimum level of the design parameter. The confirmation experiment is conducted by setting the machining parameter at the optimum level. The predicted DFRG $(\hat{\gamma})$ at its optimum level of the machining parameter can be found out by

$$
\hat{\gamma}=\gamma_{\mathrm{m}}+\sum_{i=1}^{q}\left(\bar{\gamma}_{l}-\gamma_{\mathrm{m}}\right)
$$

where, $\gamma_{\mathrm{m}}$ is mean of DFRGs all experiments run, $\bar{\gamma}_{l}$ mean of DFRG at the optimum level of its parameter, and $q$ is the number of machining parameter that significantly affect DFRG. For the quality improvement, the initial machining parameter is considered to be $\mathrm{I}_{\mathrm{P}}=4 \mathrm{~A}$, Ton $=100 \mu \mathrm{s}, \mathrm{Vg}=55 \mathrm{~V}, \Gamma=60 \%$ 
and $\mathrm{Pc}=5 \mathrm{~g} / \mathrm{l}$. Table 5 shows the confirmatory experiment at this optimum level. The optimum parameter for this experiment is obtained from the main effect plot. DFRG value for initial machining condition is 0.537 , and it enhances up to 0.582 for optimal design. So the improvement of DFRG is 0.045 . From this investigation, it is clearly seen that quality characteristics are greatly improved.

\section{Table 5}

Results of machining performance using initial and optimal machining parameters

\begin{tabular}{|c|c|c|c|}
\hline & \multirow[t]{2}{*}{ Initial machining parameters } & \multicolumn{2}{|c|}{ Optimum machining parameter } \\
\hline & & Prediction & Experiment \\
\hline Setting level & $\mathrm{IP}_{1} \mathrm{~T}_{\text {on2 }} \mathrm{V}_{\mathrm{g} 2} \Gamma_{2} \mathrm{Pc}_{2}$ & $\mathrm{IP}_{1} \mathrm{~T}_{\text {on}} 3 \mathrm{~V}_{\mathrm{g}} \Gamma_{2} \mathrm{Pc}_{3}$ & $\mathrm{IP}_{1} \mathrm{~T}_{\text {on } 3} \mathrm{~V}_{\mathrm{g} 3} \Gamma_{3} \mathrm{Pc}_{3}$ \\
\hline MRR & 2.3708 & & 2.4591 \\
\hline TWR & 0.03198 & & 0.01066 \\
\hline $\mathrm{R}_{\mathrm{a}}$ & 6.330 & & 5.124 \\
\hline DFRG & 0.537 & 0.776 & 0.582 \\
\hline Improvement in DFRG & $8.37 \%$ & & \\
\hline
\end{tabular}

\section{Conclusions}

In this research work, a hybrid optimization technique (desirability coupled with the fuzzy-logic method) has been performed to optimize the PMEDM responses viz. material removal rate, tool wear rate and surface roughness during machining of AISI 304 stainless steel. Based on the experiment the following conclusions are drawn:

- The optimum parametric combination of peak current $=4 \mathrm{~A}$, pulse on time $=150 \mu \mathrm{s}$, gap voltage $=65 \mathrm{~V}$, duty cycle $=65 \%$ and powder concentration $=10 \mathrm{~g} / 1$ has been determined.

- ANOVA results show that peak current is the most significant parameter for all the responses.

- A hybrid technique of desirability-fuzzy logic method in combination with RSM-based experimental design has a good potential to do away with the difficult task of multi response optimization by converting the data into a single DFRG. Thus it can be effectively used for optimizating the machining parameter in PMEDM to enhance the material removal rate and surface finish with lower tool wear rate.

\section{References}

Acilar, A. M., \& Arslan, A. (2011). Optimization of multiple input-output fuzzy membership functions using clonal selection algorithm. Expert Systems with Applications, 38(3), 1374-1381.

Barzani, M. M., Zalnezhad, E., Sarhan, A. A., Farahany, S., \& Ramesh, S. (2015). Fuzzy logic based model for predicting surface roughness of machined $\mathrm{Al}-\mathrm{Si}-\mathrm{Cu}-\mathrm{Fe}$ die casting alloy using different additives-turning. Measurement, 61, 150-161.

Bhaumik, M., \& Maity, K. P. (2014). Study the effect of Tungsten carbide electrode on stainless steel (AISI 304) Material in die sinking EDM. Journal of Material Science and Mechanical Engineering, 1(1), 1-6.

Bhaumik, M., \& Maity, K. P. (2015). Multi response optimization of machining parameters in electro discharge machining of AISI 304 using desirability with Grey relational analysis. International Journal of Pure and Applied Research in Engineering and Technology, 3(9), 15-22.

Bhaumik, M., \& Maity, K. P. (2016). Multi-response optimization by using the hybrid technique in electro discharge machining of AISI 304. International Journal of Engineering Research in Africa, 26, 68-75.

Dewangan, S., Gangopadhyay, S., \& Biswas, C. K. (2015). Multi-response optimization of surface integrity characteristics of EDM process using grey-fuzzy logic-based hybrid approach. Engineering Science and Technology, an International Journal, 18(3), 361-368. 
Dewangan, S., Gangopadhyay, S., \& Biswas, C. K. (2015). Study of surface integrity and dimensional accuracy in EDM using Fuzzy TOPSIS and sensitivity analysis. Measurement, 63, 364-376.

Latha, B., \& Senthilkumar, V. S. (2009). Analysis of thrust force in drilling glass fiber-reinforced plastic composites using fuzzy logic. Materials and Manufacturing Processes, 24(4), 509-516.

Latha, B., \& Senthilkumar, V. S. (2010). Modeling and analysis of surface roughness parameters in drilling GFRP composites using fuzzy logic. Materials and Manufacturing Processes, 25(8), 817827.

Montgomery, D.C. (2001). Design and Analysis of Experiments, 5th ed., John Wiley and Sons, Inc.

Pandey, A., \& Singh, S. (2010). Current research trends in variants of Electrical Discharge Machining: A review. International Journal of Engineering Science and Technology, 2(6), 2172-2191.

Pandey, A. K., \& Dubey, A. K. (2012). Taguchi based fuzzy logic optimization of multiple quality characteristics in laser cutting of Duralumin sheet. Optics and Lasers in Engineering, 50(3), 328-335.

Pandey, R. K., \& Panda, S. S. (2015). Optimization of bone drilling using Taguchi methodology coupled with fuzzy based desirability function approach. Journal of Intelligent Manufacturing, 26(6), 1121-1129.

Rajagopal, S. P., Ganesh, V., Lanjewar, A. V., \& Sankar, M. R. (2013). Past and current status of hybrid electric discharge machining (H-EDM) processes. Advanced Materials Manufacturing \& Characterization, 3(1), 111-118.

Ramanujam, R., Maiyar, L. M., \& Vasan, K. V. M. (2014). Multi Response Optimization Using ANOVA And Desirability Function Analysis: A Case Study In End Milling Of Inconel Alloy. ARPN Journal of Engineering and Applied Sciences, 9(3), 457-463.

Reddy, V. V., Reddy, C. S., \& Valli, P. M. (2015). Optimization of Process Parameters of Surfactant and Graphite Powder Mixed Dielectric EDM through Taguchi-Grey Relational Analysis. Bonfring International Journal of Industrial Engineering and Management Science, 5(4), 175.

Singh, A., Datta, S., Mahapatra, S. S., Singha, T., \& Majumdar, G. (2013). Optimization of bead geometry of submerged arc weld using fuzzy based desirability function approach. Journal of Intelligent Manufacturing, 24(1), 35-44.

Rupajati, P., Soepangkat, B.O.P., Pramujati, B., \& Agustin, H.C.K. (2014). Optimization of recast layer thickness and surface roughness in the wire EDM process of AISI H13 tool steel using Taguchi and fuzzy logic. Applied Mechanics and Materials, 493, 529-534.

Shabgard, M. R., Badamchizadeh, M. A., Ranjbary, G., \& Amini, K. (2013). Fuzzy approach to select machining parameters in electrical discharge machining (EDM) and ultrasonic-assisted EDM processes. Journal of Manufacturing Systems, 32(1), 32-39.

Shivakoti, I., Kibria, G., Diyaley, S., \& Pradhan, B. B. (2013). Multi-objective optimization and analysis of electrical discharge machining process during micro-hole machining of D3 die steel employing salt mixed de-ionized water dielectric. Journal of Computational \& Applied Research in Mechanical Engineering (JCARME), 3(1), 27-39.

Snoeys, R., Staelens, F., \& Dekeyser, W. (1986). Current trends in non-conventional material removal processes. CIRP Annals-Manufacturing Technology, 35(2), 467-480.

Singh, S., \& Yeh, M. F. (2012). Optimization of abrasive powder mixed EDM of aluminum matrix composites with multiple responses using gray relational analysis. Journal of materials engineering and performance, 21(4), 481-491.

Tripathy, S., \& Tripathy, D. K. (2016). Multi-attribute optimization of machining process parameters in powder mixed electro-discharge machining using TOPSIS and grey relational analysis. Engineering Science and Technology, an International Journal, 19(1), 62-70.

Tang, L., \& Du, Y. T. (2014). Multi-objective optimization of green electrical discharge machining Ti$6 \mathrm{Al}-4 \mathrm{~V}$ in tap water via Grey-Taguchi method. Materials and Manufacturing Processes, 29(5), $507-513$



(C) 2017 by the authors; licensee Growing Science, Canada. This is an open access article distributed under the terms and conditions of the Creative Commons Attribution (CC-BY) license (http://creativecommons.org/licenses/by/4.0/). 